\title{
Trapped electrons and ions as particle detectors
}

\author{
Daniel Carney, ${ }^{1}$ Hartmut Häffner, ${ }^{2}$ David C. Moore, ${ }^{3}$ and Jacob M. Taylor ${ }^{4,5}$ \\ ${ }^{1}$ Physics Division, Lawrence Berkeley National Laboratory, Berkeley, CA \\ ${ }^{2}$ Department of Physics, University of California, Berkeley, CA \\ ${ }^{3}$ Wright Laboratory, Yale University, New Haven, CT \\ ${ }^{4}$ Joint Quantum Institute, National Institute of Standards and Technology, Gaithersburg, MD \\ ${ }^{5}$ Joint Center for Quantum Information and Computer Science, University of Maryland, College Park, MD
}

(Dated: August 6, 2021)

\begin{abstract}
Electrons and ions trapped with electromagnetic fields have long served as important highprecision metrological instruments, and more recently have also been proposed as a platform for quantum information processing. Here we point out that these systems can also be used as highly sensitive detectors of passing charged particles, due to the combination of their extreme charge-tomass ratio and low-noise quantum readout and control. In particular, these systems can be used to detect energy depositions many orders of magnitude below typical ionization scales. As illustrations, we suggest some applications in particle physics. We outline a non-destructive time-of-flight measurement capable of sub-eV energy resolution for slowly moving, collimated particles. We also show that current devices can be used to provide competitive sensitivity to models where ambient dark matter particles carry small electric millicharges $\ll e$. Our calculations may also be useful in the characterization of noise in quantum computers coming from backgrounds of charged particles.
\end{abstract}

Detection of moving electrically charged particles is a ubiquitous problem in physics. Most charged particle detectors are based on ionization or scintillation of a detector medium, leading to detection thresholds on the order of eV. In the past few decades, semiconducting and superconducting devices based on lower energy excitations have been proposed with thresholds down to the meV range 1-4. However, for particles that interact via long-range forces like the Coulomb force, the predominant signal may occur in the center-of-mass or other collective mode of an object, which can have extremely low energies. As an example, several of us have recently proposed [5] and demonstrated [6] the direct monitoring of impulses delivered to the motion of a macroscopic mass as a pathway toward ultra-low threshold detection in optomechanical systems [7].

Here we point out that analogous techniques can be applied to electrical interactions of charged particles with single trapped ions and electrons 8 10. The achievable detection thresholds can be below the $\mu \mathrm{eV}$ scale. Since the electric force is long-ranged, even a single electron can offer substantial interaction cross-sections at sufficiently low detector thresholds, leading to remarkable detection reach. We focus primarily on electrons, since their extreme charge-to-mass ratio (at least $10^{3}$ higher than any ion) means that they set a fundamental sensitivity floor achievable with the known particles of nature. However, the basic results carry over to ions by simply rescaling the electron mass $m_{e} \rightarrow m_{\text {ion. }}$. We also note that the same results would apply to any other long-range forces coupling to these systems, not just electromagnetism.

Electrons confined in Penning traps 8, 10, have a long history in metrology, notably in precision measurement of the electron magnetic moment [11, 12. Electrons in both Penning and radio-frequency (rf) Paul traps have also been proposed as qubit systems which could enable very fast gate times in quantum computing [13[15, with single-electron trapping recently achieved experimentally in a room-temperature rf Paul trap [16]. This offers the exciting possibility of utilizing quantum information techniques to enhance the sensitivity of the device [17, 18].

As an example highlighting the extreme sensitivity of these devices, we consider the application of a singleelectron sensor to searches for dark matter candidates with small electric charge $q \ll e[1923$. Such "millicharged" dark matter (mDM) can arise in models with weak mixing between the photon and hidden-sector forces 24, and has received significant recent interest to possibly explain 22, 25, 26 an anomalous measurement in the $21 \mathrm{~cm}$ emission of cosmological hydrogen by the EDGES collaboration [27. We show that a single electron in a typical trap configuration, utilizing only ground-state cooling and single-quantum ("singlephonon") readout, would be capable of searching novel parameter space in viable scenarios in which $\sim 1 \%$ of dark matter consists particles with charges $\sim 1 \%$ of the electron charge.

Trapped electrons as impulse detectors. Both Penning and Paul traps produce electron (or ion) motion which is well-described by harmonic oscillations. Penning traps use a static, axial magnetic field combined with a static electric quadrupole potential to trap the electrons. Paul traps instead do not use a magnetic field but rather a combination of a static electric quadrupole potential and rapidly varying drive field. Time-averaging over the drive produces dynamical stability. Electron motion in the Penning trap is characterized by the axial, cyclotron, and magnetron frequencies $\left(\omega_{z}, \omega_{c}, \omega_{m}\right)$; Paul traps are characterized by three frequencies $\omega_{z}, \omega_{x} \approx \omega_{y}$ as well as the 
micromotion drive frequency $\Omega$. In either case, the frequencies are tunable; in Penning traps $\omega_{m}<\omega_{z}<\omega_{c}$.

We propose to exploit quantum control and readout of the electron motion as a sensitive impulse detector. Consider cooling the motion on an axis of our choice, with frequency $\omega$, to near the ground state $|0\rangle$. Suppose a rapid impulse is then delivered to the electron, imparting energy $\Delta E$. If $\Delta E \gtrsim \omega$, energy levels above the ground state will be populated. If some event, for example a particle collision, transfers momentum $\Delta p$ to the electron, this will impart energy $\Delta E=\Delta p^{2} / 2 m_{e}$. Thus, the fundamental detection threshold of a single-electron detector is of order

$$
\Delta p_{\mathrm{SQL}}=\sqrt{2 \hbar m_{e} \omega} .
$$

This is precisely the "standard quantum limit" (SQL) for momentum detection using a harmonic oscillator [7, 28, 29]. In terms of energy transfer, (1) translates to a detection threshold simply given by the trap frequency. For electrons trapped in the $10 \mathrm{MHz}-10 \mathrm{GHz}$ range, this corresponds to threshold energies around neV- $\mu \mathrm{eV}$, far below ionization levels. With ions, these frequencies can be down to the $10 \mathrm{~s}$ of $\mathrm{kHz}$ (for example [30]), which would produce similar momentum thresholds (1) as the increased mass competes with the decreased frequency.

The essential duty cycle of a detector of this nature would proceed in three steps. First, we prepare the motion near the ground state $|0\rangle$. The system then evolves for some time $\Delta t$. After $\Delta t$ has passed, we estimate the change in momentum. A detector "click" occurs if this momentum change is greater than the threshold (1) 31.

We remark that the fundamental assumption we make in what follows is simply that we can measure changes in momentum at or near the SQL in a bandwidth similar to $\omega$. Thus, both the preparation and measurement elements can be relaxed to include higher temperature initial states and/or less effective measurement, so long as near SQL-limited momentum measurement is achieved. This has been demonstrated, for example, using both non-demolition [12] and projective [32] measurement of the oscillator level. In addition to achieving measurement errors at SQL levels, the primary experimental requirement is that the heating rate (in phonons per second $\left.\Gamma_{Q}\right)$ should be small. Defining the quantum quality factor $Q=\omega / \Gamma_{Q}$, our duty cycle is bounded by $\Delta t \lesssim Q / \omega$, so that we will not obtain spurious clicks due to heating.

We substantiate these generic requirements by examining the cryogenic electron Penning trap in [12, in which the $150 \mathrm{GHz}$ cyclotron mode was monitored via quantum non-demolition measurements. This device satisfies all of the above requirements, and has $Q$ sufficiently high such that the effective "duty cycle" defined above can be on the order of days. Scaling this setup to lower-frequency modes (to lower the detection threshold (1D) should be feasible. In a typical cryogenic ion trap with a $d \sim 50 \mu \mathrm{m}$ spacing between the ion and the electrodes, the phonon

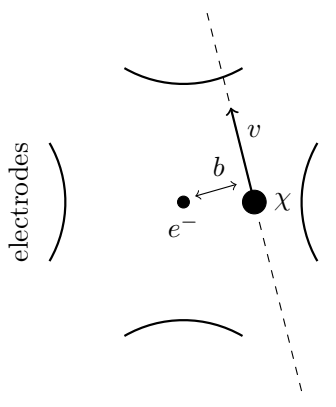

FIG. 1. Kinematics of a scattering event in a Paul trap. The charged particle $\chi$ impinges on the trapped electron with impact parameter $b$ and velocity $v$.

heating rates for modes around $1 \mathrm{MHz}$ are of order a few phonons per second 33, 34, with a corresponding quantum $Q \gtrsim 10^{5}$. These rates should scale roughly as $1 / d^{4}$ [35] and linearly in $m_{e} / m_{\text {ion }}$, so to achieve a rate of a few phonons per day would require building a trap with $d=\mathcal{O}(\mathrm{cm})$. Typical dimensions of Penning traps approach this size and indeed the BASE collaboration finds heating rates of 2 quanta/day for the $18 \mathrm{MHz}$ cyclotron motion of an anti-proton stored in a cryogenic Penning trap with $d=1.8 \mathrm{~mm} \mathrm{[36].}$

Charged particle detection. We now specialize to the case of a passing electrically charged particle $\chi$ of mass $m_{\chi}$ and charge $q_{\chi}$. The Coulomb force between this and the electron has magnitude $F=\lambda / r^{2}$. We will use "natural" units $\hbar=c=e=1$, so $\lambda=\alpha q_{\chi}$ in terms of the fine structure constant $\alpha$.

Let $b$ denote the impact parameter between $\chi$ and the electron and $v$ the relative initial velocity (see Figure 1). We can define a "fly-by time" $\tau=b / v$, during which the majority of the impulse is transferred. The simplest case, which we will assume here, is that $\tau \ll \omega_{i}^{-1}$ for all the trap frequencies (including, in the Paul trap, the micromotion drive frequency $\Omega$ 37]). With the typical velocities and impact parameters of interest here, this should be valid for modes with frequency below around $100 \mathrm{GHz}$. In this limit, the electron is essentially a free particle during the collision, and we have simple Rutherford scattering.

The events with the lowest momentum transfer will be at large impact parameter. This will produce small-angle scattering, for which the momentum transfer is

$$
\Delta p=\frac{\lambda}{b v} .
$$

We note that (2) is true even for relativistic velocities $v \approx c$ [38], although for sufficiently low velocity it breaks down because the incoming particle will not have enough kinetic energy.

We can combine (1) and (2) to estimate an effective cross-sectional area, $\sigma_{\text {eff }}$, through which a passing particle will be detectable above threshold. Setting these 
equal and solving for the impact parameter gives

$$
\begin{aligned}
\sigma_{\mathrm{eff}} & =4 \pi b^{2}=4 \pi x_{0}^{2} \frac{\lambda^{2}}{v^{2}} \\
& \approx 40 \mathrm{~nm}^{2} \times \frac{q_{\chi}^{2}}{v^{2}} \times\left(\frac{1 \mathrm{GHz}}{\omega}\right),
\end{aligned}
$$

where $x_{0}^{2}=1 / 2 m_{\mathrm{e}} \omega$ is the size of the ground-state wavefunction of the electron. These cross-sections are sufficiently large to allow even a single trapped ion or electron to detect interesting fluxes of charged particles.

These estimates assume that the momentum transfer due to scattering is entirely delivered to the mode of interest (say, the axial mode). For the Coulomb interaction, this will be the case only if the particle $\chi$ is incoming with velocity transverse to this axis. Thus if we know the direction of the incoming particles, this alignment is the optimum configuration. If we are instead trying to detect particles with unknown or random incoming directions, we will be sensitive only to a fraction of incoming particles

$$
f_{A}(\Delta p)=\sqrt{1-\Delta p_{\mathrm{th}}^{2} / \Delta p^{2}}
$$

This natural (and tunable) anisotropy enables directional sensitivity of the detector and is thus a feature in applications like dark matter searches.

As an illustration, Figure 2 shows the expected sensitivity for a trapped electron or ion to a given flux of background electrons. These could could arise for example from cosmogenic or radiogenic particles (e.g. the $\sim 10^{3} \mathrm{~cm}^{-2}$ day $^{-1}$ astrophysical muon flux) hitting the trap and causing showers of slow electrons. Alternatively, local sources of radioactivity may produce secondary lowenergy particles at non-negligible fluxes, including secondary electrons or slow-moving recoiling daughter ions from decays on electrode surfaces. While such radioactivity has been considered as a possible source of errors in superconducting qubits [39, 40, here we see that this may also eventually be a consideration for trapped electron or ion based systems.

One possible application of such a detector could be to time-of-flight measurements of slowly moving charges in order to measure their kinetic energy [41, 42]. For example, one could use a retarding potential to slow electrons produced in nuclear reactions into a collimated beam. To resolve the energy of particles in the beam, one could use a coincidence measurement with a pair of trappedcharged devices spaced at distance $L$. The single-shot measurement time to resolve an SQL fluctuation is a single trap period $\Delta T=2 \pi / \omega[29$. A coincidence measurement would then be able to determine $v$ up to an error of order $\Delta v=L \Delta T / T^{2}=v^{2} \Delta T / L$. Resolving a $\chi$ particle's energy to precision $\Delta E=m_{\chi} v \Delta v$ would thus require a baseline of order $L=(2 E)^{3 / 2} \Delta T / m^{1 / 2} \Delta E$. For example, determining the energy of an $E \sim 1 \mathrm{eV}$ electron

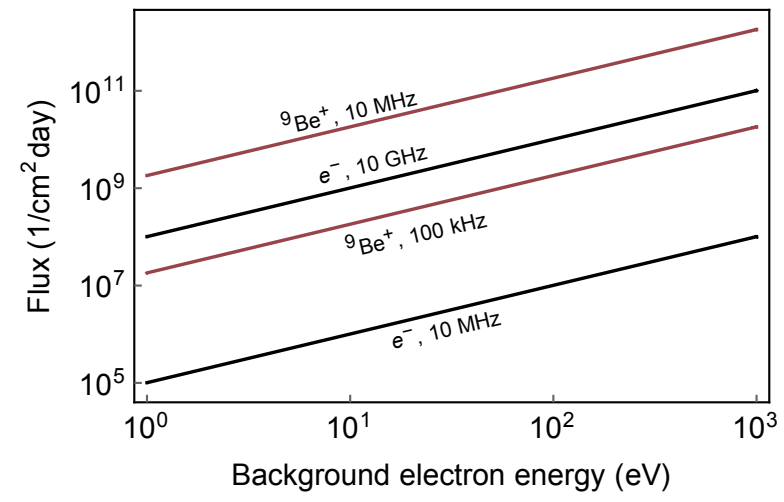

FIG. 2. Detectable levels of flux of ambient charged particles (here taken to be electrons), as a function of the ambient particle's kinetic energy. Each curve represents a trap with an electron or ion (here we use beryllium [43] to illustrate) with various frequencies. A background of electrons with the flux shown will produce impulses above threshold (1) at a rate of 1 event/day.

at a resolution of $\Delta E=1 \mathrm{eV}$ could be achieved with a pair of $100 \mathrm{MHz}$ traps spaced at $10 \mathrm{~mm}$.

Sensitivity to millicharged dark matter (mDM). As a possible application of these ideas, we now consider detection of an ambient background of dark matter particles with small charges $q_{\chi} \ll 1$. Other DM-electron couplings - for example, coupling to electron number $L_{e}$ [44, or via a massive mediator [45] - would have similar detection reach, as long as the range of the force is longer than the cross section (3).

Our detection target is thus an approximately homogeneous distribution of $\mathrm{mDM}$ particles, with positive and negative charges in arbitrary proportion. We assume these all have the same mass $m_{\chi}$. Astrophysical observations independent of the detailed nature of dark matter indicate that the average dark matter mass density should be around $0.3 \mathrm{GeV} / \mathrm{cm}^{3}$ [46], so the number density of $\mathrm{mDM}$ particles is

$$
n_{\chi}=\frac{0.3}{\mathrm{~cm}^{3}} \times f_{\mathrm{q}} \times\left(\frac{1 \mathrm{GeV}}{m_{\chi}}\right) .
$$

Here $f_{q} \lesssim 4 \times 10^{-3}[22$ is the fraction of DM which is charged. These charges will deliver random impulses on our detector. Let $f(\boldsymbol{v})$ denote their velocity distribution. The number of events, per unit time and momentum transfer, is given by the Rutherford formula

$$
\frac{d R}{d \Delta p}=n_{\chi} \frac{2 \pi \lambda^{2}}{\Delta p^{3}} \eta(\Delta p) .
$$

Here $\eta(\Delta p)=\int_{v_{\min }}^{\infty} d \boldsymbol{v} f(\boldsymbol{v}) v^{-1}$ parametrizes effects from the velocity distribution, and $v_{\min }=\Delta p / m_{e}$ is the minimum incoming velocity kinematically necessary to produce a kick $\Delta p$ to the electron [47. Integrating (6), multiplied by (4) for single-axis monitoring, from $\Delta p=\Delta p_{\text {th }}$ 

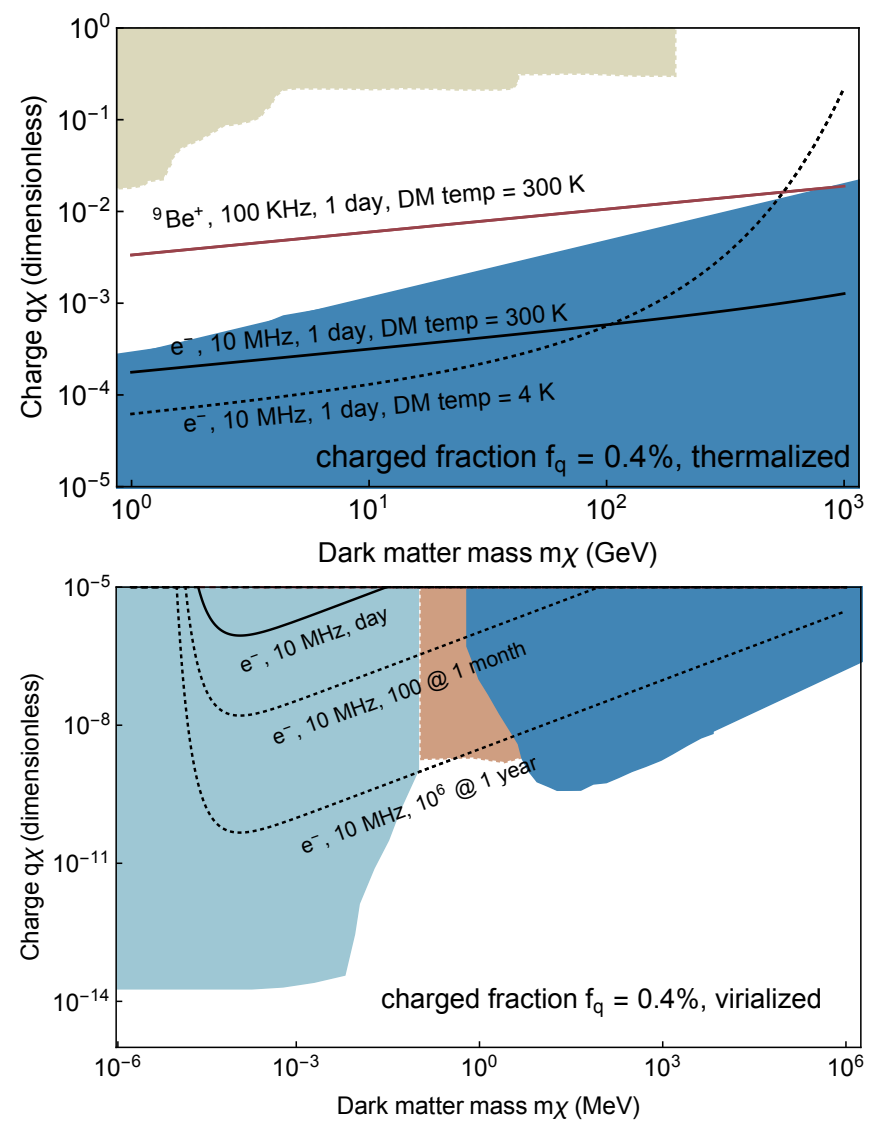

FIG. 3. Projected millicharged dark matter sensitivity with various trap frequencies and integration times. We consider detection along only a single trap axis (4). Top plot: highcharge DM thermalized at room temperature [300 K] (or at liquid He temperature [4 K]). Bottom: low-charge DM virialized to the galaxy. Currently excluded parameter space is shaded: bounds in light blue come from stellar astrophysics 48, 49, dark brown from cosmology (BBN and CMB $N_{\text {eff }}$ [48, 50]), dark blue from direct detection experiments 45], and light brown from a variety of collider experiments 51 . 54 .

upwards will produce the total number of events, per unit time, above the detection threshold $\Delta p_{\text {th }}$.

For electrically charged DM, the details of the distribution present at a terrestrial experiment are poorly understood, since these charges interact with astrophysical magnetic fields, the atmosphere, etc. [19, 22, 55]. In particular, at sufficiently large charge, mDM may lose substantial energy when transiting the atmosphere and earth en route to an experiment [23, 56, 57. Such energy loss explains the lack of direct detection limits above $q_{\chi} \gtrsim 10^{-4}$ [45], because the subsequently slowed $\mathrm{mDM}$ will have kinetic energy below existing dark matter detection thresholds based on ionization [58 60] or excitation of electron-hole pairs in $\mathrm{Si} 6163$. In contrast, the substantially lower thresholds possible with trapped electrons, as well as the the $v^{-2}$ enhancement in the cross section (3), could enable detection in the currently unconstrained region of parameter space above the direct detection constraints around $10^{-5} \lesssim q_{\chi} \lesssim 10^{-1}$.

In Figure 3 we present a pair of example sensitivities in these two different charge regions. For charges $q \gtrsim 10^{-5}$ at relatively heavy mass $m_{\chi} \gtrsim 1 \mathrm{GeV}, \mathrm{DM}$ is expected to be captured and thermalized into the Earth [56]. For smaller charges, in contrast, the DM should be virialized with the galaxy. In both plots we use the highly conservative number density (5) with $f_{q}=0.4 \%$; in particular, the number density for captured and thermalized mCPs may be orders of magnitude larger [56]. Even with these conservative assumptions, we see that a single electron or ion monitored for a day has substantial detection reach in novel parameter space.

Substantial improvements in cross-section could be reached with either multiple charges in a single trap, multiple traps, or a combination, as in quantum computing. For example, current ion traps can support crystals of $\gtrsim 10^{2}$ charges in a single trap spaced by their mutual Coulomb repulsion at $\gtrsim \mu \mathrm{m}$ distances 64. To illustrate the requirements to achieve sensitivity below the lower edge of cosmological constraints at $q_{\chi} \lesssim 10^{-10}$, we also show the sensitivity with $10^{6}$ electrons 65 .

We note two key advantages of this type of detection. One is the inherent directional sensitivity. As described above, the trap has tunable anisotropy between the different axes, so directional sensitivity is possible for single events (by measuring multiple axes) or statistically with many events (by looking for temporal variations in the event rate in a single axis). Second, a number of traditional backgrounds are suppressed by the effective velocity discrimination in (3).

Such background sources include the atmospheric muon flux described above, charged particles originating from plateout of Rn daughters (including from long-lived species such as ${ }^{210} \mathrm{~Pb}$ ) on detector surfaces, and natural radioactivity such as $\beta$ s from ${ }^{40} \mathrm{~K}$ in detector materials. These background sources have been studied in detail by rare-event searches for dark matter and $0 \nu \beta \beta$ decay 66 68, and can be mitigated by material selection and handling to produce primary event rates sub-dominant to atmospheric muons. As described above in the context of qubit errors, production of low energy secondary charged particles remains an open question. Such secondaries have been identified in precision experiments sensitive to sources of low-energy $e^{-}$such as KATRIN [6971. Nonetheless, given the small trap geometries, it is expected that it is feasible to reject a substantial fraction of such backgrounds by surrounding the traps with traditional charged particle detectors and vetoing coincidences with high-energy interactions. Furthermore, the use of multiple electrons and searching for "tracks" would enable robust rejection of many backgrounds [5]. For these reasons, and the very low-event data from existing traps [12, 36, we assumed a background-free search is possi- 
ble in producing Figure 3 although more detailed study would be necessary in an experimental realization.

In a very practical sense, our approach is to look for a sudden change in momentum due to an "event", when compared to stochastic and quantum noise backgrounds. The SQL is a reasonable starting point for the limits of this type of detection. We mention however that advanced techniques (e.g., using motional Fock states [72, squeezing [73, or spin entanglement [74]) can push measurements beyond this limit.

Conclusions. Single electrons have the highest chargeto-mass ratio known in nature. Like atoms, electrons are universal - each has exactly the same propertiesand can be controlled and read out at the single-phonon level. Thus single-electron systems provide a fundamental platform for detection of tiny impulses, well below typical ionization energies. As a simple example, we showed that just one to a few trapped electrons can be used to perform novel searches for millicharged dark matter. Beyond the millicharged scenario, these systems can enable ultra low-threshold detection to any putative new long-range force coupling to the electron or ion. With trapped ions and electrons emerging as a platform for quantum information processing, these systems (with either one or many charges) also provide an opportunity to test quantum-enhanced protocols for sensing.

Acknowledgements. We thank Nikita Blinov, Gordan Krnjaic, Zhen Liu, Sam McDermott, Nadav Outmezguine, Ryan Plestid, Trey Porto, and Steve Rolston for discussions. DC is supported by the US Department of Energy under contract DE-AC02-05CH11231 and Quantum Information Science Enabled Discovery (QuantISED) for High Energy Physics grant KA2401032. $\mathrm{HH}$ acknowledges support from AFOSR through grant FA9550-20-1-0162, the NSF QLCI program through grant number OMA-2016245. DCM is supported, in part, by NSF Grant PHY-1653232 and the HeisingSimons Foundation.

[1] S. A. Hertel, A. Biekert, J. Lin, V. Velan, and D. N. McKinsey, "Direct detection of sub-gev dark matter using a superfluid ${ }^{4}$ He target," Phys. Rev. D 100, 092007 (2019)

[2] S. Griffin, S. Knapen, T. Lin, and K. M. Zurek, "Directional Detection of Light Dark Matter with Polar Materials," Phys. Rev. D 98, 115034 (2018), arXiv:1807.10291 [hep-ph]

[3] Y. Hochberg, I. Charaev, S.-W. Nam, V. Verma, M. Colangelo, and K. K. Berggren, "Detecting subgev dark matter with superconducting nanowires," Phys. Rev. Lett. 123, 151802 (2019)

[4] Y. Hochberg, Y. Zhao, and K. M. Zurek, "Superconducting detectors for superlight dark matter," Phys. Rev. Lett. 116, 011301 (2016).

[5] D. Carney, S. Ghosh, G. Krnjaic, and J. M. Taylor,
"Proposal for gravitational direct detection of dark matter," Phys. Rev. D 102, 072003 (2020), arXiv:1903.00492 [hep-ph].

[6] F. Monteiro, G. Afek, D. Carney, G. Krnjaic, J. Wang, and D. C. Moore, "Search for composite dark matter with optically levitated sensors," Phys. Rev. Lett. 125, 181102 (2020), arXiv:2007.12067 [hep-ex].

[7] D. Carney, G. Krnjaic, D. C. Moore, C. Regal, et al., "Mechanical Quantum Sensing in the Search for Dark Matter," Quantum Sci. Technol. 6, 024002 (2021), arXiv:2008.06074 [physics.ins-det].

[8] D. Wineland, P. Ekstrom, and H. Dehmelt, "Monoelectron oscillator," Phys. Rev. Lett. 31, 1279 (1973).

[9] W. Paul, "Electromagnetic traps for charged and neutral particles," Rev. Mod. Phys. 62, 531 (1990).

[10] H. Dehmelt, "Experiments on the structure of an individual elementary particle," Science 247, 539-545 (1990).

[11] D. Hanneke, S. Fogwell, and G. Gabrielse, "New measurement of the electron magnetic moment and the fine structure constant," Phys. Rev. Lett. 100, 120801 (2008).

[12] D. Hanneke, S. F. Hoogerheide, and G. Gabrielse, "Cavity control of a single-electron quantum cyclotron: Measuring the electron magnetic moment," Phys. Rev. A 83, 052122 (2011).

[13] G. Ciaramicoli, I. Marzoli, and P. Tombesi, "Scalable quantum processor with trapped electrons," Phys. Rev. Lett. 91, 017901 (2003).

[14] I. Marzoli et al., "Experimental and theoretical challenges for the trapped electron quantum computer," Journal of Physics B: Atomic, Molecular and Optical Physics 42, 154010 (2009).

[15] N. Daniilidis, D. J. Gorman, L. Tian, and H. Häffner, "Quantum information processing with trapped electrons and superconducting electronics," New Journal of Physics 15, 073017 (2013).

[16] C. Matthiesen, Q. Yu, J. Guo, A. M. Alonso, and H. Häffner, "Trapping electrons in a room-temperature microwave paul trap," Phys. Rev. X 11, 011019 (2021).

[17] V. Giovannetti, S. Lloyd, and L. Maccone, "Quantumenhanced measurements: beating the standard quantum limit," Science 306, 1330-1336 (2004).

[18] C. J. Riedel, "Direct detection of classically undetectable dark matter through quantum decoherence," Phys. Rev. D 88, 116005 (2013).

[19] S. D. McDermott, H.-B. Yu, and K. M. Zurek, "Turning off the lights: how dark is dark matter?" Phys. Rev. D 83, 063509 (2011).

[20] A. D. Dolgov, S. L. Dubovsky, G. I. Rubtsov, and I. I. Tkachev, "Constraints on millicharged particles from planck data," Phys. Rev. D 88, 117701 (2013)

[21] K. K. Boddy, V. Gluscevic, V. Poulin, E. D. Kovetz, M. Kamionkowski, and R. Barkana, "Critical assessment of cmb limits on dark matter-baryon scattering: New treatment of the relative bulk velocity," Phys. Rev. D 98, 123506 (2018).

[22] R. Barkana, N. J. Outmezguine, D. Redigolo, and T. Volansky, "Strong constraints on light dark matter interpretation of the edges signal," Phys. Rev. D 98, 103005 (2018).

[23] T. Emken, R. Essig, C. Kouvaris, and M. Sholapurkar, "Direct Detection of Strongly Interacting Sub-GeV Dark Matter via Electron Recoils," JCAP 09, 070 (2019), arXiv:1905.06348 [hep-ph].

[24] B. Holdom, "Two u(1)'s and $\epsilon$ charge shifts," Phys. Lett. 
B 166, 196 - 198 (1986).

[25] R. Barkana, "Possible interaction between baryons and dark-matter particles revealed by the first stars," Nature 555, 71-74 (2018).

[26] A. Berlin, D. Hooper, G. Krnjaic, and S. D. McDermott, "Severely constraining dark-matter interpretations of the 21-cm anomaly," Phys. Rev. Lett. 121, 011102 (2018).

[27] J. D. Bowman, A. E. E. Rogers, R. A. Monsalve, T. J. Mozdzen, and N. Mahesh, "An absorption profile centred at 78 megahertz in the sky-averaged spectrum," Nature 555, 67-70 (2018).

[28] D. Mozyrsky, I. Martin, and M. B. Hastings, "Quantumlimited sensitivity of single-electron-transistor-based displacement detectors," Phys. Rev. Lett. 92, 018303 (2004).

[29] A. A. Clerk, "Quantum-limited position detection and amplification: A linear response perspective," Phys. Rev. B 70, 245306 (2004).

[30] G. Poulsen and M. Drewsen, "Adiabatic cooling of a single trapped ion," (2012), arXiv:1210.4309

[31] We note that a detector of this type can be calibrated by a variety of methods. The most straightforward would be to use laser pulses of known shape [6]; another would be to use a charged particle source (possibly with retarding potential to produce a slow beam).

[32] K. G. Johnson, B. Neyenhuis, J. Mizrahi, J. D. WongCampos, and C. Monroe, "Sensing atomic motion from the zero point to room temperature with ultrafast atom interferometry," Phys. Rev. Lett. 115, 213001 (2015).

[33] J. Chiaverini and J. M. Sage, "Insensitivity of the rate of ion motional heating to trap-electrode material over a large temperature range," Phys. Rev. A 89, 012318 (2014), arXiv:1310.4385

[34] J. A. Sedlacek, J. Stuart, D. H. Slichter, C. D. Bruzewicz, R. McConnell, J. M. Sage, and J. Chiaverini, "Evidence for multiple mechanisms underlying surface electric-field noise in ion traps," Phys. Rev. A 98, 63430 (2018), arXiv:1809.07761.

[35] K. R. Brown, J. Chiaverini, J. M. Sage, and H. Häffner, "Materials challenges for trapped-ion quantum computers," Nature Reviews Materials , 1-14 (2021)

[36] M. J. Borchert, P. E. Blessing, J. A. Devlin, J. A. Harrington, T. Higuchi, J. Morgner, C. Smorra, E. Wursten, M. Bohman, M. Wiesinger, A. Mooser, K. Blaum, Y. Matsuda, C. Ospelkaus, W. Quint, J. Walz, Y. Yamazaki, and S. Ulmer, "Measurement of ultralow heating rates of a single antiproton in a cryogenic penning trap," Phys. Rev. Lett. 122, 043201 (2019)

[37] When $\tau$ is larger than $\Omega^{-1}$ but smaller than the inverse trapping frequencies $\omega_{i}^{-1}$ there may be important corrections to scattering due to the micromotion of the system, but we will assume Mathieu parameter $q_{i} \ll 1$, consistent with current experiments, and neglect this correction in what follows. We also note that axial detection will be insensitive to micromotion.

[38] The 1st edition of Jackson's Electrodynamics had a nice discussion of this (see his equation 13.1), but this material seems to have been removed in subsequent editions.

[39] A. Vepsäläinen et al., "Impact of ionizing radiation on superconducting qubit coherence," Nature 584, 551-556 (2020), arXiv:2001.09190 [quant-ph].

[40] M. McEwen et al., "Resolving catastrophic error bursts from cosmic rays in large arrays of superconducting qubits," (2021), arXiv:2104.05219 [quant-ph].
[41] M. Jerkins, J. R. Klein, J. H. Majors, F. Robicheaux, and M. G. Raizen, "Using cold atoms to measure neutrino mass," New Journal of Physics 12, 043022 (2010).

[42] N. Steinbrink, V. Hannen, E. L. Martin, R. G. H. Robertson, M. Zacher, and C. Weinheimer, "Neutrino mass sensitivity by mac-e-filter based time-of-flight spectroscopy with the example of katrin," New Journal of Physics 15, 113020 (2013).

[43] M. J. Biercuk, H. Uys, J. W. Britton, A. P. VanDevender, and J. J. Bollinger, "Ultrasensitive detection of force and displacement using trapped ions," Nature Nanotechnology 5, 646-650 (2010).

[44] A. Arvanitaki, P. W. Graham, J. M. Hogan, S. Rajendran, and K. Van Tilburg, "Search for light scalar dark matter with atomic gravitational wave detectors," Phys. Rev. D 97, 075020 (2018), arXiv:1606.04541 [hep-ph]

[45] T. Emken, R. Essig, C. Kouvaris, and M. Sholapurkar, "Direct detection of strongly interacting sub-gev dark matter via electron recoils," Journal of Cosmology and Astroparticle Physics 2019, 070 (2019).

[46] J. Bovy and S. Tremaine, "On the local dark matter density," The Astrophysical Journal 756, 89 (2012).

[47] T. Lin, "Tasi lectures on dark matter models and direct detection," (2019), arXiv:1904.07915 [hep-ph]

[48] C. Boehm, M. J. Dolan, and C. McCabe, "A lower bound on the mass of cold thermal dark matter from planck," JCAP 2013, 041 (2013).

[49] J. H. Chang, R. Essig, and S. D. McDermott, "Supernova 1987a constraints on sub-gev dark sectors, millicharged particles, the qcd axion, and an axion-like particle," Journal of High Energy Physics 2018, 1-45 (2018).

[50] H. Vogel and J. Redondo, "Dark radiation constraints on minicharged particles in models with a hidden photon," Journal of Cosmology and Astroparticle Physics 2014, 029 (2014).

[51] A. Prinz et al., "Search for millicharged particles at slac," Phys. Rev. Lett. 81, 1175 (1998).

[52] G. Magill, R. Plestid, M. Pospelov, and Y.-D. Tsai, "Millicharged particles in neutrino experiments," Phys. Rev. Lett. 122, 071801 (2019).

[53] R. Acciarri et al., "Improved limits on millicharged particles using the argoneut experiment at fermilab," Phys. Rev. Lett. 124, 131801 (2020).

[54] A. Ball et al., "Search for millicharged particles in protonproton collisions at $\mathrm{s}=13$ tev," Phys. Rev. D 102, 032002 (2020).

[55] L. Chuzhoy and E. W. Kolb, "Reopening the window on charged dark matter," JCAP 2009, 014 (2009).

[56] M. Pospelov and H. Ramani, "Earth-bound Milli-charge Relics," (2020), arXiv:2012.03957 [hep-ph].

[57] G. Afek, F. Monteiro, J. Wang, B. Siegel, S. Ghosh, and D. C. Moore, "Limits on the abundance of millicharged particles bound to matter," (2020), arXiv:2012.08169 [hep-ex]

[58] J. Angle et al. (XENON10), "A search for light dark matter in XENON10 data," Phys. Rev. Lett. 107, 051301 (2011), [Erratum: Phys.Rev.Lett. 110, 249901 (2013)], arXiv:1104.3088 [astro-ph.CO]

[59] E. Aprile et al. (XENON), "Low-mass dark matter search using ionization signals in XENON100," Phys. Rev. D 94, 092001 (2016), [Erratum: Phys.Rev.D 95, 059901 (2017)], arXiv:1605.06262 [astro-ph.CO].

[60] P. Agnes et al. (DarkSide), "Constraints on Sub-GeV Dark-Matter-Electron Scattering from the DarkSide- 
50 Experiment," Phys. Rev. Lett. 121, 111303 (2018) arXiv:1802.06998 [astro-ph.CO]

[61] O. Abramoff et al. (SENSEI), "SENSEI: DirectDetection Constraints on Sub-GeV Dark Matter from a Shallow Underground Run Using a Prototype Skipper-CCD," Phys. Rev. Lett. 122, 161801 (2019), arXiv:1901.10478 [hep-ex].

[62] A. Aguilar-Arevalo et al. (DAMIC Collaboration), "Constraints on light dark matter particles interacting with electrons from damic at snolab," Phys. Rev. Lett. 123, 181802 (2019)

[63] D. W. Amaral et al. (SuperCDMS), "Constraints on low-mass, relic dark matter candidates from a surfaceoperated SuperCDMS single-charge sensitive detector," Phys. Rev. D 102, 091101 (2020), arXiv:2005.14067 [hepex].

[64] E. Jordan, K. A. Gilmore, A. Shankar, A. Safavi-Naini, J. G. Bohnet, M. J. Holland, and J. J. Bollinger, "Near ground-state cooling of two-dimensional trappedion crystals with more than 100 ions," Phys. Rev. Lett. 122, 053603 (2019)

[65] While this may seem like a large number, note that this is an order of magnitude fewer qubits than required to factor a number relevant to RSA encryption with Shor's algorithm [?].

[66] D. S. Akerib et al. (LZ), "The LUX-ZEPLIN (LZ) radioactivity and cleanliness control programs," Eur. Phys. J. C 80, 1044 (2020), arXiv:2006.02506 [physics.ins-det]

[67] N. Abgrall et al., "The Majorana Demonstrator radioas- say program," Nucl. Instrum. Meth. A 828, 22-36 (2016), arXiv:1601.03779 [physics.ins-det].

[68] D. S. Leonard et al., "Trace radioactive impurities in final construction materials for EXO-200," Nucl. Instrum. Meth. A 871, 169-179 (2017), arXiv:1703.10799 [physics.ins-det]

[69] F. Fränkle et al., "Radon induced background processes in the KATRIN pre-spectrometer," Astropart. Phys. 35, 128-134 (2011), arXiv:1103.6238 [physics.ins-det].

[70] F. Fränkle et al., "KATRIN background due to surface radioimpurities," (2020), arXiv:2011.05107 [physics.insdet].

[71] M. Aker et al., "First direct neutrino-mass measurement with sub-eV sensitivity," (2021), arXiv:2105.08533 [hepex]

[72] D. M. Meekhof, C. Monroe, B. E. King, W. M. Itano, and D. J. Wineland, "Generation of nonclassical motional states of a trapped atom," Phys. Rev. Lett. 76, 1796 (1996).

[73] S. C. Burd, R. Srinivas, J. J. Bollinger, A. C. Wilson, D. J. Wineland, D. Leibfried, D. H. Slichter, and D. T. C. Allcock, "Quantum amplification of mechanical oscillator motion," Science 364, 1163-1165 (2019).

[74] K. A. Gilmore, J. G. Bohnet, B. C. Sawyer, J. W. Britton, and J. J. Bollinger, "Amplitude sensing below the zero-point fluctuations with a two-dimensional trappedion mechanical oscillator," Phys. Rev. Lett. 118, 263602 (2017). 